\title{
I Year Surgical Outcomes and Safety of Combined Trans Pars Plana Vitrectomy with Iris Fixated Posterior Chamber Intraocular Lens for Subluxated Intraocular Lens and Cataracts
}

\author{
Valencia Hui Xian Foo (D) \\ Tiffany Sin Hui Bong' \\ Andrew Tsai ${ }^{1,2}$ \\ Laurence Shen Lim ${ }^{1,2}$ \\ Shamira Asith Perera ${ }^{1,2}$ \\ 'Department of Ophthalmology, \\ Singapore Eye Research Institute, \\ Singapore National Eye Centre, \\ Singapore; ${ }^{2}$ Eye-Academic Clinical \\ Program, Duke-NUS Medical School, \\ National University of Singapore, \\ Singapore
}

\begin{abstract}
Importance/Background: To describe the characteristics and 1-year postoperative surgical outcomes of primary combined trans pars plana vitrectomy (TPPV) with iris-fixated posterior-chamber intraocular lens (IF-PCIOL) implantation for subluxated cataracts and intraocular lenses (IOLs).

Methods: Consecutive cases of significantly subluxated cataracts and IOLs from January 2014 to May 2019 were included in this retrospective case series.

Results: A total of 103 eyes of 103 patients were included. Median age was 67.4 (interquartile range [IQR] 27-89) years. Thirty-two (31.1\%) had subluxated cataracts, while 71 (68.9\%) had subluxated IOLs. Overall median preoperative logMAR BCVA was 0.9 (IQR 0 2.5). Median $\log$ MAR BCVA improved to $0.3(0-1.9)$ and $0.2(0-1.8)$ for the subluxated cataract and IOL groups, $81.2 \%$ and $75 \%$ of eyes achieved $\log \mathrm{MAR} \leq 0.3$, and $94 \%$ and $97 \%$ achieved IOL stability at month 12, respectively. Eyes with subluxated IOLs had a significantly less myopic median SE if a new IOL was implanted compared to irisfixating the dislocated IOL.

Conclusion: Combined primary TPPV and IF-PCIOL implantation is a good surgical option for subluxated IOL or cataract without sufficient capsular support, with at least $75 \%$ achieving $\log$ MAR BCVA $\leq 0.3$ and $95 \%$ IOL stability at postoperative year 1 . In eyes with subluxated IOLs, explanting that IOL and iris-fixating a new IOL led to a reduced SE and better visual outcome at postoperative month 12 when compared to iris-fixating the dislocated IOL.
\end{abstract}

Keywords: subluxated cataracts, subluxated intraocular lenses, secondary intraocular lens fixation, iris-fixation, trans pars plana vitrectomy

\section{Introduction}

Intraocular lens (IOL) implantation with insufficient capsular support in adults is challenging. It can be achieved with anterior chamber IOL (ACIOL) or posteriorchamber IOL (PCIOL) techniques such as via iris-fixation (IF), sutured scleral, or intrascleral fixation. ${ }^{1-3}$ Angle supported ACIOLs have fallen out of favour due to the high rates of associated complications of corneal decompensation, secondary glaucoma and uveitis-glaucoma-hyphema (UGH) syndrome from the close proximity of lens haptics to the cornea, trabecular meshwork and iris. ${ }^{4,5}$ Increasingly, many surgeons tend to favour the use of a PCIOL. A subset of these patients may
Correspondence: Shamira Asith Perera Singapore National Eye Centre, II Third Hospital Avenue, 16875I, Singapore

Tel +6562277255

Fax +6562263995

Email shamira.perera@singhealth.com.sg 
require concomitant trans pars plana vitrectomy (TPPV) for subluxated crystalline lens or IOL. These eyes pose an extra challenge because of the lack of vitreous support. Combined vitrectomy surgery with IOL implantation has several advantages. The surgical goal of vitrectomy and rapid visual rehabilitation with IOL implantation can be achieved simultaneously. IOP can be kept stable by continuous infusion pressure during the operation. Furthermore, a formed and stable AC provides the surgeon with optimal visualization to perform suture fixation of the PC IOL safely and accurately.

To date, most studies have reported small case series of short follow-up periods for combined TPPV and IF-PCIOL in the same sitting after removal of subluxated lenses or IOLs with lensectomy or IOL explant respectively. ${ }^{6-12}$ Therefore, we aim to report a case series of the 1-year surgical outcomes and safety profile of TPPV and IFPCIOL implantation for subluxated cataracts and IOLs.

\section{Methods}

The study was conducted in accordance with the tenets of the Declaration of Helsinki and was carried out with the approval of the SingHealth Centralized Institutional Review Board. A waiver of consent from subjects was obtained as the study was accomplished with anonymized data in the form of coded datasets, with no personal identifying information. Patient confidentiality was maintained throughout the study. We performed a retrospective analysis of consecutive patients who underwent primary combined TPPV and iris-fixated IOL implantation surgeries for subluxated crystalline lenses or IOLs from January 2014 to June 2019 at the Singapore National Eye Centre. The cases that were included were substantially subluxated ( $\geq 4$ clock hours of zonular laxity or detachment, or with vitreous in the anterior chamber) such that an anterior segment approach would have been difficult, and might have led to a dropped IOL or nucleus eventually requiring a combined TPPV with or without a lensectomy. The utilization of a vitreoretinal (VR) surgeon together with an anterior segment (AS) surgeon was considered the best approach to attain good visual rehabilitation without risking further iatrogenic retinal complications. Exclusion criteria included: subluxated ACIOLs which needed removal, all eyes with poor visual function due to other reasons (eg amblyopia, macular scar or optic atrophy) before surgery, eyes that had other combined surgeries with TPPV/IFIOL including cornea or glaucoma surgery and eyes with substantial missing data from health records. All operations were performed by two surgeons (S.A.P. and L.L.S.). 103 eyes of 103 consecutive participants each were recruited into the study.

We collected the following data if available: demographic information, ocular comorbidities, type, date, and any complications of the original cataract surgery, predisposing factors for crystalline lens or IOL subluxation, best-corrected visual acuity (BCVA) by logMAR chart at presentation, ultrasound biomicroscopy (UBM) findings, manifest refraction, preoperative autokeratometry (AK) and axial length (AL) measurements [IOLMaster; Carl Zeiss Meditec], contact A-scan or immersion A-scan biometry. Intraoperative and postoperative complications were recorded. Refractions were converted to spherical equivalent (SE) by adding spherical power to $1 / 2$ cylinder power. Predicted refraction values were calculated using either the Sander-Retzlaff-Kraff Theoretical (SRK/T), Haigis Suite, Hoffer-Q, or Theoretic formula. Median absolute error (MAE) was the absolute difference between the actual postoperative subjective SE and predicted SE refraction, and does not consider the direction of the error. Refractive prediction error (RPE) was defined as the actual postoperative subjective SE refraction minus predicted SE refraction, and indicates the direction (sign) of the error. The primary outcomes are postoperative BCVA, SE, MAE and RPE at months 1 and 12. Secondary outcomes include IOL stability, postoperative complications, and any repeat surgeries at the same time points.

\section{Surgical Technique}

A 23-gauge TPPV was performed by a single VR surgeon (L.L.S.). Core vitrectomy was performed, with careful attention to release the subluxated cataract or IOL from the surrounding vitreous. The dislocated cataract was removed using a fragmatome, whereas IOLs were grasped with a pair of vitreoretinal forceps and brought to the anterior chamber and the capsule remnants stripped away under viscoelastic. If the dislocated IOL was to be reused we assessed the following factors for suitability: clarity of optic (ie free from discolouration, glistenings, laser pits) and whether the haptics were in good condition. The PPV trocars were removed after a search for retinal pathology and the scleral wounds were self-sealing, if not sutured with 7-0 Vicryl (Ethicon, Somerville, NJ, USA). The remainder of the procedure was performed by a single anterior segment surgeon (S.A.P.). 3 and 9 o'clock cornea incisions were made. In cases where the dislocated IOL was unsuitable for fixation and needed explantation, the 
IOL was bisected with intraocular scissors and removed via the temporal $2.65 \mathrm{~mm}$ corneal incision. A 3-piece AcrySof MA60AC IOL (Alcon Surgical, Inc, Fort Worth, TX) was implanted through the temporal corneal incision. Each haptic was placed in the ciliary sulcus whilst the optic was retained in the anterior chamber to deliver a stable optic captured-IOL with intracameral carbachol (Miostat, Alcon, Inc.). The PCIOL was sutured to the iris near the 6 and 12 o'clock positions using a modified McCannel technique. One end of a 10-0 polypropylene suture (Prolene; Ethicon, Somerville, NJ, USA) on a double armed spatulated needle (CTC-6; Ethicon, Somerville, NJ, USA) was passed through clear cornea through the midperipheral iris and then underneath the haptic of the 3-piece IOL. The pass was completed back through the iris and clear cornea on the opposite side. A limbal paracentesis was made to externalise the suture ends using a Kuglen hook (AH0670, MST, Redmond, WA) and the sutures were tied securely together with a 3,1,1 formation and cut short so as not to abrade the corneal endothelium. This was performed twice on each haptic to achieve a 4-point fixation. Finally, a Sinskey hook pushed the optic behind the iris, the remaining viscoelastic was removed and stability and centration of the IOL were reassessed. Subconjunctival dexamethasone and antibiotics were administered.

\section{Statistical Analysis}

The Shapiro-Wilk test was used to assess for normality of our data distribution, which was found to be non-normal ( $p$ $<0.05)$. We used the Mann-Whitney test to determine the median BCVA (measured in logMAR), absolute sphere, cylinder, SE, MAE and RPE (measured in dioptres [D]) at all time points and their differences between the two groups. Data is expressed as the median value (interquartile range [IQR 25 to 75 percentile]). Chi-square test was used to analyse categorical variables. Analyses were performed using SPSS version 21 (SPSS Inc. Chicago, IL, USA).

\section{Results}

One hundred and three eyes of 103 consecutive patients were included in this study. The median age of the study cohort was 63 (age range 27-89) years old, with no significant difference in age between the subluxated cataract and IOL group. 49 (47.6\%) were male. 32 (31.1\%) patients presented with subluxated cataracts while 71 (68.9\%) had subluxated IOLs.

\section{Subluxated Cataracts}

The most common identifiable risk factor for subluxated cataracts in our cohort was pseudoexfoliation syndrome (6.3\%), followed by Marfan's syndrome (3.1\%) and trauma $(3.1 \%)$. Most patients presented with pseudophacodonesis $(56.3 \%)$, followed by a dropped cataract onto the retina $(31.3 \%)$ and dislocation into the anterior chamber $(21.8 \%)$. The median preoperative VA was logMAR 0.9 (0 to 2.5) (Table 1).

At month 1, 22 eyes (68.8\%) were available at the follow-up. Median logMAR BCVA had significantly improved to $0.3(0-1.8)(\mathrm{p}<0.04)$ with a median SE of $-0.6(-1.8$ to -0.4$) \mathrm{D}$, and $100 \%$ of IOLs were stable and well-centered. The most common early postoperative complications at month 1 were intraocular pressure (IOP) spike $>24 \mathrm{mmHg}$ at $18.2 \%$, followed by retinal detachment $(4.5 \%)$, persistent inflammation $(4.5 \%)$ and optic capture (4.5\%). No patient experienced postoperative suprachoroidal haemorrhage or endophthalmitis (Table 2).

At month 12, 16 eyes $(50 \%)$ were available at the follow-up. Median logMAR BCVA was 0.2 (0-1.9) (Table 2). $81.2 \%$ of eyes had achieved a logMAR BCVA better or equal to 0.30 . $93.8 \%$ of eyes had stable and wellcentered IOLs, with $6.3 \%$ of eyes ( 1 eye) having recurrent subluxation of the IOL. The subject underwent a repeat uncomplicated iris fixation of IOL with new sutures, yielding $\log$ MAR BCVA of 0 at postoperative month 6 from the second iris-fixation. The most common late postoperative complications at month 12 were intraocular pressure (IOP) spike $>24 \mathrm{mmHg}$ at $12.5 \%$, followed by persistent inflammation $(6.3 \%)$ and delayed presentation of hyphema with vitreous hemorrhage $(6.3 \%)$ with high IOP eventually needing an anterior chamber washout. $75 \%$ of eyes were able to achieve a SE within \pm 0.5 of predicted refraction, and $93.8 \%$ were able to achieve a SE within \pm 1.0 of predicted refraction (Table 3 ).

\section{Subluxated IOL}

The most common identifiable risk factor for subluxated IOLs was previous complicated cataract surgery, with 39 eyes $(54.9 \%)$ that had a dropped nucleus and 5 eyes (7\%) with posterior capsular rupture (PCR). Other common identifiable risk factors for subluxation were previous TPPV (8.5\%) and trauma (2.8\%). Of the 9 eyes with previous TPPV, 3 were for retinal detachments, 2 had dislocated sulcus IOL, 1 had a dropped nucleus, 1 had vitreous haemorrhage $(\mathrm{VH})$ and 1 had epiretinal 
Table I Overall Demographic and Ocular Characteristics of Patients with Subluxated Cataract/Intraocular Lens (IOL) in the Study at Baseline

\begin{tabular}{|c|c|c|c|c|}
\hline & All & $\begin{array}{l}\text { Subluxated Cataract } \\
\text { (Median or n/lQR or \%) }\end{array}$ & $\begin{array}{c}\text { Subluxated IOL } \\
\text { (Median or } n / I Q R \text { or } \\
\% \text { ) }\end{array}$ & $\mathbf{p}$ \\
\hline & $N=103$ & $\mathbf{N}=32$ & $\mathbf{N}=7 \mathbf{I}$ & \\
\hline $\begin{array}{l}\text { Median age at original cataract diagnosis, } \\
\text { years (IQR) }\end{array}$ & $63(58-69)$ & $57(5 \mid-63)$ & $66(59-72)$ & 0.07 \\
\hline \multicolumn{5}{|l|}{ Ethnicity } \\
\hline Chinese & 73 (70.9) & $27(84.4)$ & $46(64.8)$ & 0.58 \\
\hline Malay & $24(23.3)$ & $4(12.5)$ & $20(28.2)$ & \\
\hline Indian & $6(5.8)$ & I (3.I) & $5(7.0)$ & \\
\hline Gender & & & & 0.65 \\
\hline Female & $54(52.4)$ & $18(56.2)$ & $26(36.6)$ & \\
\hline Male & $49(47.6)$ & $14(43.8)$ & $35(49.4)$ & \\
\hline \multicolumn{5}{|l|}{ Type of original cataract operation } \\
\hline Phacoemulsification & - & - & $34(47.9)$ & \\
\hline ECCE & - & - & $3(4.2)$ & \\
\hline ICCE & - & - & I (I.4) & \\
\hline Unknown & - & - & $33(46.5)$ & \\
\hline Complicated original cataract surgery & & & & - \\
\hline PCR with no nucleus drop & $5(6.8)$ & - & $5(7.0)$ & \\
\hline PCR with nucleus drop & $39(37.9)$ & - & $39(54.9)$ & \\
\hline Unknown & - & - & $27(38.1)$ & \\
\hline $\begin{array}{l}\text { Identifiable predisposing factors for } \\
\text { subluxation }\end{array}$ & & & & 0.005 \\
\hline Trauma & $3(2.9)$ & I (3.I) & $2(2.8)$ & \\
\hline Previous TPPV & $6(5.8)$ & $0(0)$ & $6(8.5)$ & \\
\hline Pseudoexfoliation syndrome & $2(1.9)$ & $2(6.3)$ & $0(0)$ & \\
\hline Marfan's syndrome & I (0.9) & I (3.I) & $0(0)$ & \\
\hline Retinitis pigmentosa & I (0.9) & $0(0)$ & I (I.4) & \\
\hline $\begin{array}{l}\text { Years after original cataract extraction } \\
\text { before subluxation/median (IQR) }\end{array}$ & - & - & $6(0.5-40)$ & \\
\hline Classification of Subluxation & & & & $<0.001$ \\
\hline Dislocation into Anterior Chamber & $9(8.7)$ & $7(2 \mid .8)$ & $3(4.2)$ & \\
\hline Pseudophacodonesis & $36(35.0)$ & I8 (56.3) & $20(28.2)$ & \\
\hline $\begin{array}{l}\text { Posterior dislocation into the anterior } \\
\text { vitreous cavity }\end{array}$ & $16(15.5)$ & $4(12.5)$ & $12(16.9)$ & \\
\hline Trap door-like dislocation & $12(11.7)$ & $2(6.3)$ & $9(12.6)$ & \\
\hline Dropped on the retina & $30(29.1)$ & I (3I.3) & $28(38.4)$ & \\
\hline Preoperative logMAR BCVA (median/IQR) & $0.9(0-2.5)$ & $0.9(0-2.5)$ & $0.9(0-2.0)$ & 0.20 \\
\hline Axial Length, mm (median/IQR) & $24.6(21.9-24.4)$ & $24.1(22.2-27.5)$ & $25.2(21.9-24.4)$ & 0.72 \\
\hline
\end{tabular}

Abbreviations: IQR, interquartile range; ECCE, extracapsular cataract extraction; ICCE, intracapsular cataract extraction; PCR, posterior capsule rupture; TPPV, trans pars plana vitrectomy; BCVA, best corrected visual acuity.

membrane peel. For the remaining eyes, the cause of the subluxated IOL or lens could not be determined definitively by historical inquiry or by ocular examination. Most patients presented with a dropped IOL onto the retina $(38.4 \%)$, pseudophacodonesis $(28.2 \%)$, and posterior dislocation into the anterior vitreous $(16.9 \%)$. The median preoperative VA was $\operatorname{logMAR} 0.9$ (0 to 2) (Table 1). 
Table 2 Post-Operative Surgical Outcomes and Complications of Subluxated Cataracts and IOLs

\begin{tabular}{|c|c|c|c|c|}
\hline & All & $\begin{array}{l}\text { Subluxated Cataract } \\
\text { (Median or } n / l Q R \text { or \%) }\end{array}$ & $\begin{array}{c}\text { Subluxated IOL } \\
\text { (Median or } n / l Q R \text { or \%) }\end{array}$ & $\mathbf{p}$ \\
\hline \multicolumn{5}{|l|}{ Post-operative Month I } \\
\hline No. of eyes with follow-up & $85(82.5)$ & $22(68.8)$ & $63(88.7)$ & \\
\hline LogMAR BCVA /median (IQR) & $0.2(0-1.8)$ & $0.3(0-1.8)$ & $0.2(0-1.8)$ & 0.29 \\
\hline Sphere (D) /median (IQR) & $0(-6.3-2.0)$ & $0(-0.9-0)$ & $-0.3(-3.8-2.0)$ & 0.66 \\
\hline Cyl (D) /median (IQR) & $-1(-3.5-0.8)$ & $-0.8(-3.5-0.8)$ & $-1.3(-3.5-0)$ & 0.21 \\
\hline SE (D) /median (range) & $-0.9(-4.8-1.9)$ & $-0.6(-1.8$ to -0.4$)$ & $-1.0(-4.8-1.4)$ & 0.32 \\
\hline IOL status & & & & 0.34 \\
\hline Stable & $84(98.9)$ & $22(100.0)$ & $62(98.4)$ & \\
\hline Complications & & & & 0.74 \\
\hline IOP spike $>24 \mathrm{~mm} \mathrm{Hg}$ & $13(15.3)$ & $4(18.2)$ & $9(14.3)$ & \\
\hline Persistent inflammation & $3(3.5)$ & I (4.5) & $2(3.2)$ & \\
\hline CMO & I (I.2) & $0(0)$ & I (I.6) & \\
\hline Hyphema/VH & $2(2.4)$ & $0(0)$ & $2(3.2)$ & \\
\hline Retinal detachment & $\mathrm{I}(\mathrm{I} .2)$ & I (4.5) & $0(0)$ & \\
\hline Tilted IOL & I (I.2) & $0(0)$ & I (I.6) & \\
\hline Optic capture & $2(2.4)$ & I (4.5) & I (I.6) & \\
\hline \multicolumn{5}{|l|}{ Post-operative Month I 2} \\
\hline No. of eyes with follow-up & $48(46.6)$ & $16(50.0)$ & $32(45.1)$ & \\
\hline LogMAR BCVA/median (IQR) & $0.2(0-1.9)$ & $0.2(0-1.9)$ & $0.2(0-1.8)$ & 0.70 \\
\hline Sphere (D) /median (IQR) & $0(-2.5-1.8)$ & $0.1(-0.5-0.8)$ & $0(-2.5-1.8)$ & 0.71 \\
\hline Cyl (D)/median (IQR) & $-1.5(-4.5$ to -0.3$)$ & $-1.8(-2.5$ to -1.23$)$ & $-1.4(-4.5$ to -0.3$)$ & 0.76 \\
\hline SE (D) & $-0.7(-2.6-0.1)$ & $-1.0(-1.3-0.1)$ & $-0.6(-2.9-0)$ & 0.58 \\
\hline IOL status & & & & 0.37 \\
\hline Stable & $46(95.8)$ & $15(93.8)$ & $31(96.9)$ & \\
\hline Subluxated & $2(4.2)$ & I (6.3) & I (3.I) & \\
\hline Complications & & & & 0.33 \\
\hline IOP spike $>24 \mathrm{~mm} \mathrm{Hg}$ & $6(12.5)$ & $2(12.5)$ & $4(12.5)$ & \\
\hline Persistent inflammation & $I(2.1)$ & I (6.3) & $0(0)$ & \\
\hline CMO & $2(4.2)$ & I (6.3) & I (3.I) & \\
\hline Hyphema/VH & $3(6.3)$ & I (6.3) & $2(6.3)$ & \\
\hline PBK & $2(4.2)$ & $0(0)$ & $3(9.4)$ & \\
\hline Retinal detachment & I (2.I) & I (6.3) & $0(0)$ & \\
\hline Optic capture & $0(0)$ & $0(0)$ & I (3.I) & \\
\hline Tilted IOL & I (2.I) & $0(0)$ & I (3.I) & \\
\hline No. with logMAR BCVA $\leq 0.30$ & 37 (77.1) & $13(81.2)$ & $24(75.0)$ & 0.56 \\
\hline No. of eyes with improvement in BCVA & $38(79.2)$ & $13(8 \mid .2)$ & $25(78.1)$ & 0.47 \\
\hline
\end{tabular}

Abbreviations: BCVA, best corrected visual acuity; IQR, interquartile range; D, dioptre; IOL, intraocular lens; IOP, intraocular pressure; CMO, cystoid macular oedema; $\mathrm{VH}$, vitreous haemorrhage; SE, spherical equivalent; PBK, pseudophakic bullous keratopathy.

At month 1, 63 eyes $(88.7 \%)$ were available at the follow-up (Table 2). Median logMAR BCVA had significantly improved to $0.2(0-1.8)(\mathrm{p}<0.01)$ with a median SE of $-1.0(-4.8-1.4)$ D. $98.4 \%$ of IOLs were stable and well-centered. The most common early postoperative complications at month 1 were IOP spike $>24 \mathrm{mmHg}$ at $14.3 \%$, hyphema/vitreous haemorrhage (3.2\%), persistent inflammation $(3.2 \%)$ cystoid macular oedema [CMO] (1.6\%). 1 IOL (1.6\%) was tilted, and $1(1.6 \%)$ had optic capture. The eye with optic capture was managed conservatively as it was still centered within the visual axis and had spontaneous resolution of optic capture by month 12 . No patient experienced postoperative suprachoroidal haemorrhage or endophthalmitis. 
Table 3 Comparison of Refractive Outcomes at Month 12

\begin{tabular}{|l|c|c|c|c|}
\hline & All & $\begin{array}{c}\text { Subluxated Cataract } \\
\text { (Median or n/lQR or \%) }\end{array}$ & $\begin{array}{c}\text { Subluxated IOL } \\
\text { (Median or n/lQR or \%) }\end{array}$ & $\begin{array}{c}\mathbf{P} \\
\mathbf{N}=\mathbf{3 2}\end{array}$ \\
\cline { 2 - 5 } & $\mathbf{N}=\mathbf{4 8}$ & $\mathbf{N}=\mathbf{~ 1 6}$ & $-0.20(-2.2-0.2)$ & 0.45 \\
Predicted Refraction (SE) & $-0.2(-2.2-0.2)$ & $-0.2(-0.6-0)$ & $-1.0(-1.3-0.1)$ & 0.58 \\
Postoperative subjective refraction (SE) & $-0.7(-2.6-0.1)$ & $-0.6(-2.9--0.2)$ & $-0.8(-1.4-0.2)$ & 0.39 \\
Refractive prediction error (SE) & $-0.5(-2.2-0.2)$ & $-0.4(-1.0--0.1)$ & $0.3(0.1-0.5)$ & 0.28 \\
Median absolute error (SE) & $0.3(0.1-0.5)$ & $0.2(0.0-0.3)$ & $20(62.5)$ & 0.32 \\
Number within \pm 0.50 SE [n (\%)] & $32(66.7)$ & $12(75.0)$ & $29(90.6)$ & 0.29 \\
Number within \pm I.0 SE [n (\%)] & $44(91.6)$ & $15(93.8)$ & & \\
\hline
\end{tabular}

Abbreviations: SE, spherical equivalent; IQR, interquartile range.

At month 12, 32 eyes (45.1\%) were available at the follow-up (Table 2). Median logMAR BCVA was $0.2(0-$ 1.8). $75 \%$ of eyes had achieved a logMAR BCVA better or equal to $0.30 .96 .9 \%$ of eyes had stable and wellcentered IOLs, with $3.1 \%$ ( 1 eye) having recurrent subluxation of the IOL. The subject also underwent a repeat uncomplicated iris fixation of IOL with new sutures. The most common late postoperative complications at month 12 were IOP spike $>24 \mathrm{mmHg}$ at $12.5 \%$, followed by pseudophakic bullous keratopathy [PBK] (9.4\%), with 2 eyes requiring Descemet membrane endothelial keratoplasty (DMEK). 1 eye had optic capture $(3.1 \%)$ that required pupilloplasty and repair of iridodialysis with IOL repositioning. $62.5 \%$ of eyes were able to achieve a SE within \pm 0.5 of predicted refraction, and $90.6 \%$ was able to achieve a SE within \pm 1.0 of predicted refraction (Table 3).

In the subluxated IOL group, comparing between eyes which reused the pre-existing IOL to those with a new IOL implanted, the latter group had a significantly lower median SE at months 1 and 12 and better $\log$ MAR BCVA at month 12. At postoperative month 1, median SE were -1.4 and -0.5 respectively $(p<0.01)$ with median BCVA of 0.2 and 0.1 respectively $(p=0.26)$. At postoperative month 12 , the median SE were -0.9 and -0.5 respectively $(\mathrm{p}<$ 0.01 ) while the median BCVA was 0.3 and 0.2 respectively $(\mathrm{p}=0.026)$ (not shown in Tables).

There were no significant differences in visual outcomes between the subluxated cataract and IOL groups. The RPE of the subluxated IOL group was almost twice that of the subluxated cataract group $(-0.8[\mathrm{IQR}-1.4$ to 0.2 ] vs -0.4 [IQR -1.0 to -0.1$], \mathrm{p}=0.39)$ though the difference was not significant. MAE was also not significantly different between both group (0.3 [IQR 0.1 to 0.5$]$ ) vs 0.2 [IQR ( 0 to 0.30 respectively, $\mathrm{p}=0.28$ )] (Table 3 ).

\section{Discussion}

Our study is one of the largest case series in Asian eyes in the literature that reports the 1-year surgical outcomes and safety profile of combined TPPV and primary IF-PCIOL implantation for subluxated cataracts and IOLs. At postoperative year 1, in eyes with subluxated cataracts, the median $\operatorname{logMAR}$ BCVA was 0.3 (IQR 0-1.8), with three-quarters of subjects attaining $\log \mathrm{MAR} B C V A \leq 0.3$ and a $96 \%$ IOL stability rate. In eyes with subluxated IOLs, the median logMAR BCVA was 0.2 (IQR 0-1.8), with also three-quarters of subjects attaining $\log \mathrm{MAR}$ BCVA $\leq 0.3$ and a $97 \%$ IOL stability rate. Our results add to the current literature that this surgical approach gives favorable visual outcomes. The most common early postoperative complications were raised IOP $>24 \mathrm{mmHg}$ at $13 \%$ and persistent inflammation at $3 \%$ in both groups, while the most common late postoperative complication included raised IOP $>24 \mathrm{mmHg}$ at $12.5 \%$ and hyphema/ $\mathrm{VH}$ at $6.3 \%$ in both groups respectively, with additionally persistent inflammation (6.3\%) and $\mathrm{CMO}(6.3 \%)$ in the subluxated cataract group, and PBK (9.4\%) and CMO $(3.1 \%)$ in the subluxated IOL group.

In terms of postoperative BCVA, the median logMAR BCVA at year 1 was 0.2 in both subluxated cataract and IOL groups, with three-quarters of the cohort attaining at least $\log$ MAR BCVA of 0.3 at 1 year. These rates are similar to other published studies for both types of eyes showing that the mean postoperative $\log$ MAR ranged from 0.07 to 0.51 at month 12 up to $20 .^{6-13}$ Condon et al reported that $70 \%$ of eyes in a series of 46 eyes that underwent IF-PCIOL implantation for subluxated IOLs were able to obtain a BCVA of $\log 0.3$ or better at a mean follow-up of 24 months (range 6 to 50 months). ${ }^{14}$ When comparing results of IF-PCIOL to other methods of secondary IOL implantation, an earlier 
comparative retrospective study concluded no differences in final BCVA between transscleral-fixed IOL, IFPCIOL, and ACIOL. ${ }^{10}$ Two other studies similarly reported no significant differences in postoperative BCVA between IF-PCIOL and SFIOL eyes at $3^{15}$ and 16 months. $^{9}$ However postoperative BCVA was significantly worse in the IF-PCIOL group only at postoperative week 1 , attributing this slower visual recovery to the increased inflammatory reaction from pigments and intravascular components released from penetration of the iris stroma with a needle into the anterior chamber. Furthermore, miotics used to induce optic capture may compromise vision during the early postoperative period. We did not manage to capture the information on the trend in BCVA from postoperative day 1 to week 1 in our study which would be of value when counselling patients preoperatively.

We found a high rate of IOL stability with a wellcentered IOL of $94 \%$ in subluxated cataract eyes and $97 \%$ in subluxated IOL eyes in our series at 1 year. These stability rates were equivalent to or better than those reported in earlier studies of IF-PCIOL at 1 year, with recurrent subluxation rates ranging from $0 \%$ to $17.1 \%$ in adult eyes. ${ }^{6-11}$ In comparison to SFIOL, Kim et al reported a significant earlier recurrence rate in the IFPCIOL group compared to the SFIOL group, suggesting that the difference in recurrence rates between the two groups was due to lesser tissue solidity for the anchoring suture in the iris compared to the sclera. ${ }^{9}$

In our study, we also found the postoperative MAE greater and RPE more myopic, though not significant for both, for the subluxated IOL compared to the subluxated cataract group. Selecting a more hyperopic-powered IOL for IF-PCIOL implantation with combined TPPV in the subluxated IOL group possibly lead to a better refractive result with this technique. Brunin et al reported no significant differences in RPE between sulcus-fixed IOL with or without optic capture, ACIOL, IF-PCIOL or transscleral sutured IOL eyes. ${ }^{10}$ Furthermore, in a sub-analysis of eyes with subluxated IOL (analysis not shown), interestingly we found that using a new IOL led to a significantly less myopic SE and better BCVA outcomes at postoperative month 12, compared to eyes which reused the old IOL for iris-fixation. To our knowledge, no study to date has compared head-to-head the visual outcomes between reusing the preexisting IOL and implanting a new IOL for patients with subluxated IOL. We are unsure of the reason behind this; though this new finding needs to be confirmed by future larger studies. Furthermore, the main advantage of exchanging the dislocated IOL would be in the setting of a very large Soemmering's ring which could cause secondary pupil block if sutured to the iris.

Overall, the most common early postoperative complications were raised IOP $>24 \mathrm{mmHg}$ at $13 \%$ and persistent inflammation at $3 \%$ in both groups, while the most common late postoperative complication included raised IOP $>24 \mathrm{mmHg}$ at $12.5 \%$ and hyphema/ $\mathrm{VH}$ at $6.3 \%$ in both groups respectively, with additionally persistent inflammation $(6.3 \%)$ and $\mathrm{CMO}(6.3 \%)$ in the subluxated cataract group, and PBK $(9.4 \%)$ and CMO (3.1\%) in the subluxated IOL group. In our study, around $75 \%$ of eyes with IOP spikes $>24 \mathrm{mmHg}$ had to be continued on their topical antiglaucoma eye drops for satisfactory IOP control by postoperative month 12 . Our findings of higher rates of early and late postoperative IOP spikes are corroborated by Kim et al in Asian eyes reporting that IFPCIOL eyes tended to have higher postoperative IOP compared to the SFIOL group during the first week of surgery. ${ }^{9}$ This is attributed due to a greater inflammatory response from iris pigment release observed in Asian eyes, which have more pigmented and thicker irides in contrast to Western eyes. We noted 2 eyes in the subluxated IOL group at month 1 , and 1 eye in the subluxated cataract group with a delayed presentation of hyphema/ $\mathrm{VH}$ by month 12. Recurrent hyphema and VH are part of a rare spectrum of square-edge ACIOL designs, iris-fixated and sulcus-fixated IOLs, in addition to iridocyclitis, spontaneous micro-hyphema, and UGH syndrome. ${ }^{16}$ These haemorrhagic complications occur due to posterior iris chafing and ciliary body contact with the IOL haptics in the sulcus and have been reported to have a delayed presentation of up to 6.5 years later. ${ }^{17}$ We found a low overall rate of CMO in our study of $1.2 \%$ at 1 month and $4.2 \%$ at 12 months. The rate of early CMO was comparable if not lower than rates reported in the literature for IF-PCIOL (0 to $16.7 \%),{ }^{18}$ given the extent of iris manipulation and iridodonesis intraoperatively in a vitrectomised eye. Existing studies conducted in a mix of Western and Asian eyes for IF-PCIOL reported a slightly lower incidence of IOP spikes (1.7\% to $16.6 \%)$, hyphema/VH (2.5\%), and PBK (7.1\%) compared to our study. ${ }^{6-11}$

The overall low rate of retinal detachment in this study (2\%) can be attributed to the thorough approach to vitreous management and peripheral retinal check with indentation compared to other studies which report up to $10 \%$ when this is not utilized, ${ }^{7}$ capitalizing on the low incidence of $\mathrm{RD}$ 
from this combined approach for severely subluxated cataracts or IOLs. For the 2 eyes presenting with subluxated IOL that eventually required a DMEK for PBK, there was no prior corneal pathology of note or preoperative endothelial cell count (ECC) performed; this would highlight to anterior segment surgeons the importance of performing ECC before surgery and preoperative counselling.

Many published studies to date on PC-IFIOL describe its secondary implantation in aphakia in the absence of capsular support. ${ }^{19-22}$ Majority of studies have described combined PPV and iris fixation in dislocated IOLs. ${ }^{6-13}$ Faria et al described the combined vitrectomy and iris fixation of the same preexisting dislocated 3-piece IOL in a study cohort of 36 eyes of 36 patients and concluded that iris-fixation of the subluxated IOL gave good long term IOL stability with improved vision and minimal complications. ${ }^{11}$ Soiberman et al similarly studied combined TPPV and iris suturing of posteriorly dislocated IOLs and came to the same conclusion. ${ }^{13}$ There have been, however, only a handful of small or short-term published studies to date on the outcome of combined TPPV with IF-PCIOL for subluxated or dislocated cataracts. ${ }^{6,8}$ Sedziek-Marcinek et al described a series of secondary anterior chamber iris-claw IOL for aphakia in various indications in 132 patients, including cases with implantation during complicated cataract surgery and posterior capsule damage as well as during combined TPPV with luxated IOL extraction. The study reported a BCVA of $\log$ MAR 0.3 to 0.5 (excluding patients with concomitant penetrating keratoplasty) with stable refraction and a low percentage of complications including $4.54 \%$ of IOL subluxation, but no significant IOP rise across all groups after 4 years. ${ }^{23}$ Thus, our study is one of the largest case series in Asian eyes to our knowledge to demonstrate good visual outcomes and IOL stability of combined vitrectomy with posterior chamber IF-PCIOL for these cases. Furthermore, this technique has several advantages. Not only does it allow visual rehabilitation to commence immediately post-surgery, vitrectomy operating systems with their integrated pressurized infusion allows better intraoperative control of IOP, giving rise to a formed and stable AC allowing optimal visualization to perform iris fixation of the PCIOL safely and accurately. Althaus et al also demonstrated by endoscopy that chances of the needle passing through the ciliary sulcus accurately are greater when the eye maintains a normal IOP. $^{24}$

The limitations of our study stem from its retrospective nature, less than ideal follow-up, and high drop-out rate at postoperative month 12 and hence a short follow-up period of 1 year. We also did not collect data on pupil sizes, whiteto-white distance, pre- and postoperative ECC, the refractive status of the fellow eye, or iris thickness although we are aware that these might have a bearing on final results. We noted an overall rate of $4.2 \%$ of PBK in our cohort by postoperative year 1 but could not ascertain if these eyes had compromised endothelial function before their IFPCIOL surgery. The study also lacked a control group of secondary IF-PCIOL implantation for aphakic eyes, hence we are not able to conclusively determine if the prolonged surgical time in a combined technique would lead to a higher risk of endothelial compromise intraoperatively in comparison compared to other techniques. A randomised controlled trial would be difficult to conduct to accurately compare between various surgical techniques of secondary IOL implantations due to the small number of eyes presenting with subluxated cataracts or IOLs, and the presence of other variable factors such as differing degrees of existing zonular support and surgeon's preferred technique. Whilst we are fortunate in a tertiary centre to have the availability of a dedicated VR and AS surgeon, this combined approach may not be achievable in other centres. Similarly, our predominantly Asian series with thicker brown irides may be uniquely suitable for IF IOL and the results may not be extrapolatable to the thinner blue irides of their Caucasian counterparts. Furthermore, all surgeries were performed by the same single pair of surgeons in this case, hence the results may not be extrapolated to other surgeons, dependent on one's level of surgical experience.

In summary, the findings of our study suggest that combined PPV and IF-PCIOL implantation for subluxated cataracts or IOLs is a reliable surgical option, with more than $75 \%$ of eyes achieving logMAR BCVA of 0.3 or better vision at postoperative month 12 and an IOL stability rate of around $95 \%$ with a good safety profile in both groups. IFPCIOL remains an excellent alternative for subluxated cataracts or IOLs for eyes with preserved anterior segment anatomy amidst the armamentarium of surgical options for these eyes for IOL implantation with insufficient capsule support. In eyes with subluxated IOLs, explanting that IOL and iris fixating a new IOL led to a reduced myopic spherical equivalent and better visual outcome at month 12 when compared to iris fixating the dislocated IOL.

\section{Funding}

There is no funding to report. 


\section{Disclosures}

No conflicting relationship or financial disclosures exists for any author.

\section{References}

1. Wagoner MD, Cox TA, Ariyasu RG, Jacobs DS, Karp CL. Intraocular lens implantation in the absence of capsular support; a report by the American Academy of Ophthalmology (Ophthalmic Technology Assessment). Ophthalmology. 2003;110:840-859.

2. Hoffman RS, Snyder ME, Devgan U, Allen QB, Yeoh R, BragaMele R; ASCRS Cataract Clinical Committee. Management of the subluxated crystalline lens. J Cataract Refract Surg. 2013;39 (12):1904-1915.

3. Por YM, Lavin MJ. Techniques of intraocular lens suspension in the absence of capsular/zonular support. Surv Ophthalmol. 2005;50 (5):429-462.

4. Holt DG, Stagg B, Young J, Ambati BK. ACIOL, sutured PCIOL, or glued IOL: where do we stand? Curr Opin Ophthalmol. 2012;23 (1):62.

5. Drolsum L. Long-term follow-up of secondary flexible, open loop, anterior chamber intraocular lenses. $J$ Cataract Refract Surg. 2003;29:498-5035.

6. Zeh WG, Price FW Jr. Iris fixation of posterior chamber intraocular lenses. J Cataract Refract Surg. 2000;26:1028-1034.

7. Hirashima DE, Soriano ES, Meirelles RL, Alberti GN, Nosé W. Outcomes of iris-claw anterior chamber versus iris-fixated foldable intraocular lens in subluxated lens secondary to Marfan syndrome. Ophthalmology. 2010;117(8):1479-1485.

8. McClellan SF, Soiberman U, Gehlbach PL, Murakami PN, Stark WJ. Outcomes associated with concurrent iris-sutured intraocular lens placement and subluxated crystalline lens extraction. JAMA Ophthalmol. 2015;133(8):867-873. doi:10.1001/jamaophthalmol. 2015.1097

9. Kim KH, Kim WS. Comparison of clinical outcomes of iris fixation and scleral fixation as treatment for intraocular lens dislocation. $\mathrm{Am}$ J Ophthalmol. 2015;160(3):463-469.

10. Brunin G, Sajjad A, Kim EJ, et al. Secondary intraocular lens implantation: complication rates, visual acuity, and refractive outcomes. J Cataract Refract Surg. 2017;43(3):369-376.

11. Faria MY, Ferreira NP, Canastro M. Management of dislocated intraocular lenses with iris suture. Eur J Ophthalmol. 2017;27 (1):45-48.
12. Dzhaber D, Mustafa OM, Tian J, Cox JT, Daoud YJ. Outcomes and complications of iris-fixated intraocular lenses in cases with inadequate capsular support and complex ophthalmic history. Eye. 2020;3:1-8.

13. Soiberman U, Pan Q, Daoud Y, Murakami P, Stark WJ. Iris suture fixation of subluxated intraocular lenses. Am J Ophthalmol. 2015;159 (2):353-359.

14. Condon GP, Masket S, Kranemann C, Crandall AS, Ahmed II. Smallincision iris fixation of foldable intraocular lenses in the absence of capsule support. Ophthalmology. 2007;114(7):1311-1318.

15. Zhang H, Zhao J, Zhang LJ, et al. Comparison of iris-fixated foldable lens and scleral-fixated foldable lens implantation in eyes with insufficient capsular support. Int J Ophthalmol. 2016;9(11):1608.

16. Masket S. Pseudophakic posterior iris chafing syndrome. J Cataract Refract Surg. 1986;12(3):252-256.

17. Alniemi ST, Amin SR, Sculley L, Bakri SJ. Ultrasound biomicroscopy in pseudophakic patients with unexplained recurrent hyphema or vitreous hemorrhage. Semin Ophthalmol. 2018;33(2):260-264.

18. Hoh H, Ruprecht K, Nikoloudakis N, Palmowski A. Preliminary results following implantation of iris-suture-fixated posterior-chamber lenses. Ger J Ophthalmol. 1993;2:70-75. Pmid: 8485442

19. Chalkiadakis SE, Parikakis EA, Taylor SR. Sutureless 25-gauge pars plana vitrectomy combined with retropupillary fixation of an iris-claw intraocular lens. Case Rep Ophthalmol. 2016;7(3):433-439.

20. Riazi M, Moghimi S, Najmi Z, Ghaffari R. Secondary ArtisanVerysise intraocular lens implantation for aphakic correction in posttraumatic vitrectomized eye. Eye. 2008;22(11):1419-1424.

21. van der Meulen IJ, Gunning FP, Vermeulen MG, de Smet MD. Artisan lens implantation to correct aphakia after vitrectomy for retained nuclear lens fragments. J Cataract Refract Surg. 2004;30 (12):2585-2589.

22. Soiberman U, Pan Q, Daoud Y, Murakami P, Stark WJ. Iris suture fixation of subluxated intraocular lenses. Am J Ophthalmol. 2015;159 (2):353-359.

23. Sędziak-Marcinek B, Wylęgała A, Chełmecka E, Marcinek M, Wylęgała E. Iris-Claw intraocular lens implantation in various clinical indications: a 4-year study. J Clin Med. 2021;10(6):1199. doi: $10.3390 / \mathrm{jcm} 10061199$

24. Althaus C, Sundmacher R. Endoscopically controlled optimization of trans-scleral suture fixation of posterior chamber lenses in the ciliary sulcus. Ophthalmologie. 1993;90:317-324.
Clinical Ophthalmology

\section{Publish your work in this journal}

Clinical Ophthalmology is an international, peer-reviewed journal covering all subspecialties within ophthalmology. Key topics include: Optometry; Visual science; Pharmacology and drug therapy in eye diseases; Basic Sciences; Primary and Secondary eye care; Patient Safety and Quality of Care Improvements. This journal is indexed on PubMed
Central and CAS, and is the official journal of The Society of Clinical Ophthalmology (SCO). The manuscript management system is completely online and includes a very quick and fair peer-review system, which is all easy to use. Visit http://www.dovepress.com/ testimonials.php to read real quotes from published authors. 